\title{
Microwave-assisted synthesis of 2-acetyl-5-arylthiophenes and 4-(5-arylthiophen-2-yl)thiazoles via Suzuki coupling in water
}

\author{
Kamal M. Dawood, ${ }^{* a, b}$ Manahil B. Elamin, ${ }^{\text {a }}$ and Ahmad M. Farag ${ }^{\mathrm{a}}$ \\ ${ }^{a}$ Department of Chemistry, Faculty of Science, Cairo University, Giza 12613, Egypt \\ ${ }^{b}$ Current address: Chemistry Department, Faculty of Science, Kuwait University, P.O. Box 5969, \\ Safat 13060, Kuwait \\ E-mail: $\underline{\text { dr_dawood@yahoo.com }}$
}

DOI: http://dx.doi.org/10.3998/ark.5550190.p009.018

\begin{abstract}
2-Acetyl-5-bromothiophene and 4-(5-bromothiophen-2-yl)-2-methyl-1,3-thiazole, as deactivated bromide candidates, were prepared and used for Suzuki cross-coupling reactions with a number of aryl(hetaryl)boronic acids in water or DMF as solvents. The cross-coupling reactions were carried out under thermal heating as well as microwave irradiating conditions using a benzothiazole-based $\mathrm{Pd}(\mathrm{II})$-precatalyst. Optimization of the catalytic reaction condition was also studied.
\end{abstract}

Keywords: Thiophene, thiazole, palladium catalysis, microwave, Suzuki coupling

\section{Introduction}

Thiophene derivatives are important class of heterocyclic compounds that are widely involved in many agrochemicals and pharmaceuticals. ${ }^{1}$ They are employed in drug synthesis, for example Gabitril is an antiepilepsy drug ${ }^{2}$ and Canagliflozin is a drug for the treatment of type 2 diabetes $^{3}$ (Chart 1). In addition, thiophene-based molecules have shown numerous biological activities such as antitumor, ${ }^{4}$ analgesic, ${ }^{5}$ anti-inflammatory, ${ }^{6}$ and antibacterial against Gram-positive bacteria. $^{7}$ Suzuki-Miyaura reactions are among the most powerful tools for construction of carbon-carbon bond in organic synthesis both in industry and academia. ${ }^{8-11}$ These reactions have also been widely exploited in the synthesis of natural products, ${ }^{12,13}$ and the design of pharmaceuticals. ${ }^{14}$ The use of water as green solvent has been encouraged by the desire to create cleaner, safer, and more environmentally benign chemical processes over the past few decades. ${ }^{15-}$ ${ }^{17}$ Microwave irradiation methodology has also been receiving much interest in accelerating organic synthesis. ${ }^{18,19}$ 2-Acetyl-5-arylthiophenes were synthesized in literature via different routes such as: (1) direct arylation of 2 -acetylthiophene with bromobenzenes, ${ }^{20}$ or with 
arenediazonium chlorides; ${ }^{21}$ (2) reaction of $\beta$-chloro- $\beta$-arylacroleins with sodium sulfide and chloroacetone; $^{22}$ (3) cross-coupling of of 2-acetyl-5-halothiophene with potassium $\operatorname{aryl(heteroaryl)trifluoroborate},{ }^{23}$ or with aryl(heteroaryl)boronic acids, ${ }^{24-27}$ or with sodium tetraphenylborate, ${ }^{28}$ (4) addition of acetonitrile to 2-phenylthiophene. ${ }^{29}$ Recently, we reported the utility of several $\mathrm{Pd}(\mathrm{II})$-complexes in $\mathrm{C}-\mathrm{C}$ cross coupling reactions in water under microwave irradiation conditions, ${ }^{30-39}$ in this work; Suzuki cross-coupling reactions between two bromothiophene substrates having either activating (2-acetyl) or deactivating (2-thiazol-4-yl) moieties with variety of arylboronic acids under thermal heating as well as microwave irradiation conditions are evaluated using the benzothiazole-oxime Pd(II)-complex 1.

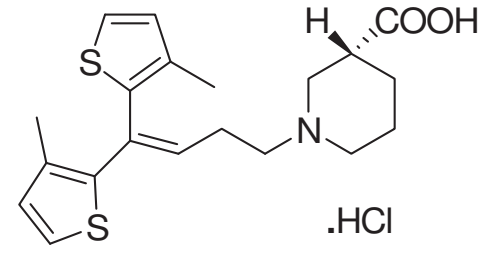

Gabitril

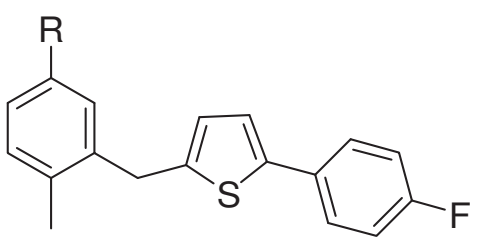

Canagliflozin

Chart 1. Thiophene containing drugs; Gabitril and Canagliflozin.

\section{Results and Discussion}

\section{Suzuki coupling of 2-acetyl-5-bromothiophene (2) with arylboronic acids under thermal heating and microwave irradiation}

The first bromide substrate; 2-acetyl-5-bromothiophene (2) was prepared by treatment of 2acetylthiophene with $\mathrm{N}$-bromosuccinimide in a mixture of glacial acetic acid and acetic anhydride according to the reported literature. ${ }^{40,41}$ Suzuki cross-coupling reactions of 2-acetyl-5bromothiophene (2) with activated and deactivated aryl(heteroaryl)boronic acids 3a-e were carried out under thermal heating as well as microwave irradiation conditions using $\mathrm{Pd}(\mathrm{II})$ precatalyst 1 as shown in Table 1. The cross-coupling reactions yielded of the corresponding 5aryl(heteroaryl)-2-acetylthiophenes 4-8 in almost full conversions with excellent isolated yields regardless of the heating mode. The reaction components molar ratios were as following; $1 \mathrm{mmol}$ 2-acetyl-5-bromothiophene (2), $1.2 \mathrm{mmol}$ arylboronic acids 3a-e, $0.6 \mathrm{mmol}$ tetrabutylammonium bromide (TBAB), 2 mmoles of $\mathrm{KOH}$ and $0.25 \mathrm{~mol} \%$ of the precatalyst 1 in water ( $3 \mathrm{~mL})$. The identity of the resulted coupling products was confirmed from their ${ }^{1} \mathrm{H}$ and ${ }^{13} \mathrm{C} \mathrm{NMR}$ and $\mathrm{MS}$ spectral data. Although, sulfur species are reported as potential catalyst poisons that cause a remarkable decrease in the catalytic activity of palladium catalysts, ${ }^{42}$ also the thiophene moiety was mentioned as poison of a perfluorinated Pd-complex, ${ }^{43}$ the cross-coupling of 2-acetyl-5bromothiophene (2) with 3-thienylboronic acid (3e) proceeded straightforward giving excellent yield of 2-acetyl-3 ,5-bithiophene (8) after 10 hours of thermal heating and after 9 min of microwave irradiation (Table 1, entry 5). 
Table 1. Suzuki coupling of 2-acetyl-5-bromothiophene (2) with arylboronic acids using $\mathrm{Pd}-$ complex 1 under thermal heating and microwave irradiation

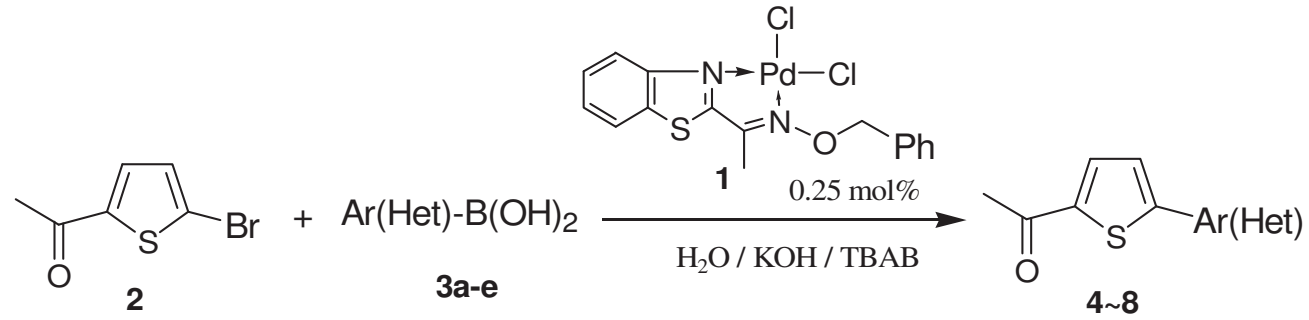

Entry $\mathrm{Ar}(\mathrm{Het})-\mathrm{B}(\mathrm{OH})_{2}$

${ }^{a}$ Reaction condition: Bromide/ boronic acid/ $\mathrm{KOH} / \mathrm{TBAB} /$ water $(3 \mathrm{~mL}): 1 / 1.2 / 2 / 0.6$, at $100{ }^{\circ} \mathrm{C}$ for recorded time. ${ }^{\text {a }}$ All values refer to the isolated yields.

\section{Synthesis of 4-(5-bromothiophen-2-yl)-2-methyl-1,3-thiazole (10)}

The hitherto unreported, 4-(5-bromothiophen-2-yl)-2-methyl-1,3-thiazole (10) was prepared, as shown in Scheme 1, using Hantzsch method involving the reaction of $\alpha$-halocarbonyl compounds with thiourea or thioamides. Thus, treatment of 2-acetyl-5-bromothiophene (2) with bromine in the presence of $\mathrm{AlCl}_{3}$ afforded 5-bromo-2-(bromoacetyl)thiophene (9) in $96 \%$ yield. $^{44}$ Reaction of 9 with thioacetamide in ethanol at reflux temperature yielded 4-(5-bromothiophen-2yl)-2-methyl-1,3-thiazole (10) in 75\% yield (Scheme 1). The structure of $\mathbf{1 0}$ was confirmed from its elemental analyses and spectroscopic data (IR, MS, ${ }^{1} \mathrm{H}$ and ${ }^{13} \mathrm{C} \mathrm{NMR}$ ) as mentioned in the experimental section. 


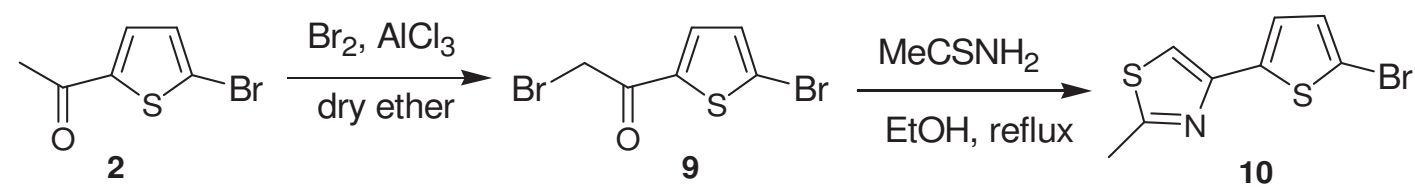

Scheme 1. Preparation of 4-(5-bromothiophen-2-yl)-2-methyl-1,3-thiazole (10).

Optimization of the catalytic condition of Suzuki coupling of 4-(5-bromothiophen-2-yl)-2methyl-1,3-thiazole (10) with phenylboronic acid under microwave irradiation

Suzuki cross-coupling of 4-(5-bromothiophen-2-yl)-2-methyl-1,3-thiazole (10) having a deactivating heterocyclic ring (thiazole) was examined. At first, it was better to optimize the catalytic condition of the new bromide substrate $\mathbf{1 0}$ with phenylboronic acid. The efficiencies of different bases and solvents as well as the proper catalyst concentration in the coupling reaction between $\mathbf{1 0}$ and phenylboronic acid were investigated under microwave irradiation. As shown in Table 2, runs $1-3$, water/TBAB/KOH catalytic reaction condition was applied under microwave irradiation at different concentrations of Pd-complex 1 (0.125, 0.25 and $0.5 \mathrm{~mol} \%$, respectively), unfortunately there was no coupled products under all these concentrations. Repeating the same reaction using a mixed solvent (DMF/water/TBAB/KOH) and $0.5 \mathrm{~mol} \%$ of Pd-complex 1 resulted in only $20 \%$ yield of $\mathbf{1 1}$. When DMF was used as a sole solvent and $\mathrm{KOH}$ as base with $0.25 \mathrm{~mol} \%$ of Pd-complex 1, 50\% yield was obtained (run 5, Table 2). Replacing $\mathrm{KOH}$ by $\mathrm{Cs}_{2} \mathrm{CO}_{3}$ using Pd-catalyst 1 in 0.25 and $0.5 \mathrm{~mol} \%$ concentrations, gave $50 \%$ and $72 \%$ yield, respectively (Table 2, runs 6,7). Raising the Pd-complex concentration into $1 \mathrm{~mol} \%$ using $\mathrm{DMF} / \mathrm{Cs}_{2} \mathrm{CO}_{3}$ resulted in full conversion as detected by TLC, after $30 \mathrm{~min}$ of microwave irradiation, with $80 \%$ isolated yield of 2-methyl-4-(5-phenythiophen-2-yl)-1,3-thiazole (11) (run 8 , Table 2). To evaluate the effect of heating mode, the cross-coupling reaction was repeated using $\mathrm{DMF} / \mathrm{Cs}_{2} \mathrm{CO}_{3}$ and $1 \mathrm{~mol} \%$ of the precatalyst 1 under thermal heating at $100 \mathrm{C}^{\circ}$ for a longer time (40 hours) where only traces of the product 11 were detected by TLC (run 9, Table 2).

Suzuki coupling of 4-(5-bromothiophen-2-yl)-2-methyl-1,3-thiazole (10) with arylboronic acids 3a-e under microwave irradiation.

Next, the optimized catalytic condition above was generalized for coupling of further arylboronic acids 3b-e with 4-(5-bromothiophen-2-yl)-2-methyl-1,3-thiazole (10) using Pd(II)-complex 1 (1 $\mathrm{mol} \%$ ) in $\mathrm{DMF} / \mathrm{Cs}_{2} \mathrm{CO}_{3}$. The reaction components molar ratios were as following; $1 \mathrm{mmol}$ of bromide 10, $1.2 \mathrm{mmol}$ of arylboronic acids 3, 2 mmoles of $\mathrm{Cs}_{2} \mathrm{CO}_{3}$ using $1 \mathrm{~mol} \%$ of the complex 1 in DMF (3 mL). The coupling reaction was conducted in a closed-vessel under microwave irradiation to give the corresponding arylthiophenylthiazole derivatives 11-14 in moderate to good isolated yields as outlined in Table 3. Unfortunately, the coupling of the bromide $\mathbf{1 0}$ with the deactivated 3-thienylboronic acid $\mathbf{3 e}$ to get the bi(thienyl)thiazole derivative $\mathbf{1 5}$ resulted in no cross-coupling reaction even after $45 \mathrm{~min}$ of microwave irradiation (entry 5, Table 3 ). 
Table 2. Optimization of the catalytic condition of Suzuki coupling of 4-(5-bromothiophen-2yl)-2-methylthiazole (10)

\begin{tabular}{ccccc} 
Run & $\begin{array}{c}\text { Cat. } \\
\text { mol\% }\end{array}$ & Solvent & Base & Yield $\%^{a}$ \\
\hline 1 & 0.125 & $\mathrm{H}_{2} \mathrm{O} / \mathrm{TBAB}$ & $\mathrm{KOH}$ & 0.0 \\
2 & 0.25 & $\mathrm{H}_{2} \mathrm{O} / \mathrm{TBAB}$ & $\mathrm{KOH}$ & 0.0 \\
3 & 0.5 & $\mathrm{H}_{2} \mathrm{O} / \mathrm{TBAB}$ & $\mathrm{KOH}$ & 0.0 \\
4 & 0.5 & $\mathrm{H}_{2} \mathrm{O} / \mathrm{DMF}(1: 2) / \mathrm{TBAB}$ & $\mathrm{KOH}$ & 20 \\
5 & 0.25 & $\mathrm{DMF}$ & $\mathrm{KOH}_{2}$ & 50 \\
6 & 0.25 & $\mathrm{DMF}$ & $\mathrm{Cs}_{2} \mathrm{CO}_{3}$ & 50 \\
7 & 0.5 & $\mathrm{DMF}$ & $\mathrm{Cs}_{2} \mathrm{CO}_{3}$ & 72 \\
8 & 1.0 & $\mathrm{DMF}$ & $\mathrm{Cs}_{2} \mathrm{CO}_{3}$ & 80 \\
9 & 1.0 & $\mathrm{DMF}$ & $\mathrm{Cs}_{2} \mathrm{CO}_{3}$ & traces \\
\hline
\end{tabular}

${ }^{a}$ Reaction condition: Bromide/ boronic acid/ base/ solvent $(3 \mathrm{~mL}): 1 / 1.2 / 2$, under microwave irradiation $\left(160{ }^{\circ} \mathrm{C}, 250 \mathrm{Watt}\right)$ for $30 \mathrm{~min} .{ }^{b}$ When repeated under thermal heating at $100{ }^{\circ} \mathrm{C}$ for $40 \mathrm{~h}$ only traces of product $\mathbf{1 1}$ were detected by TLC.

Table 3. Suzuki coupling of 4-(5-bromothiophen-2-yl)-2-methyl-1,3-thiazole (10) with arylboronic acids $\mathbf{3 a - e}$ under microwave irradiation

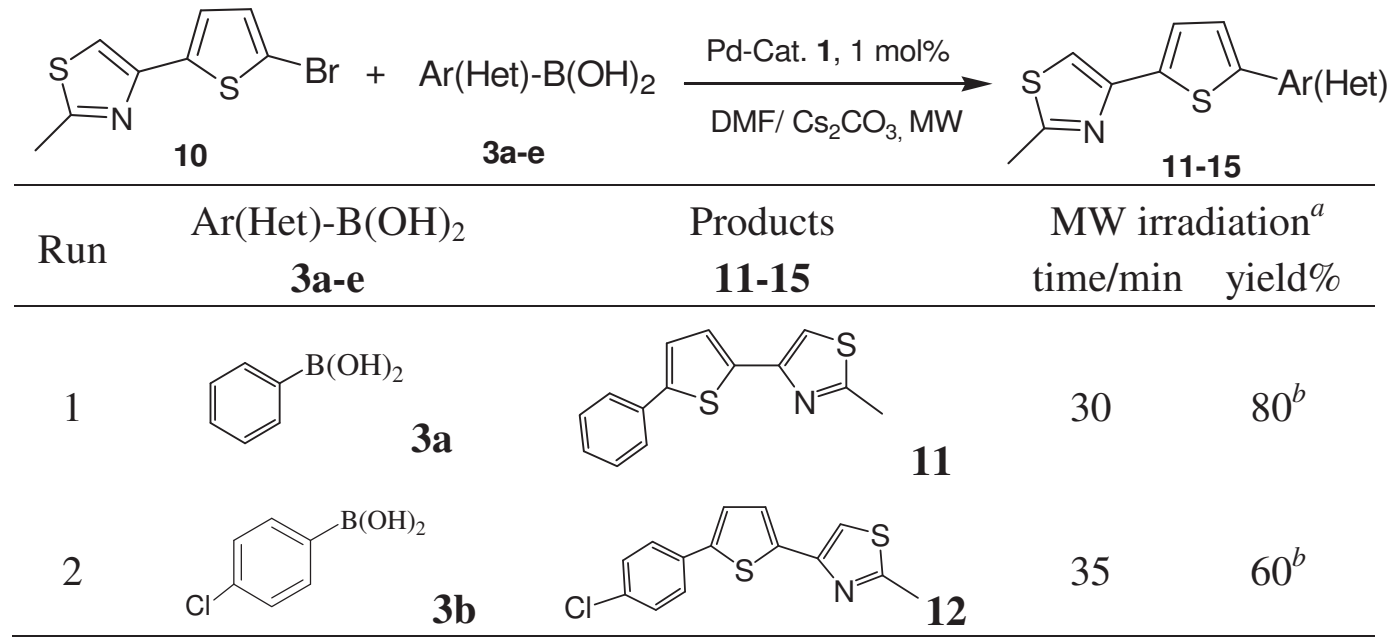


Table 3 (continued)

Run $\begin{gathered}\mathrm{Ar}(\mathrm{Het})-\mathrm{B}(\mathrm{OH})_{2} \\ \text { 3a-e }\end{gathered}$

${ }^{a}$ Conditions: Bromide 10: $1 \mathrm{mmol}$; arylboronic acids 3a-e: $1.2 \mathrm{mmol}$; $\mathrm{Cs}_{2} \mathrm{CO}_{3:} 2 \mathrm{mmol}$; DMF (3 $\mathrm{mL})$; Pd-complex 1: $1 \mathrm{~mol} \%$, microwave irradiation $\left(160{ }^{\circ} \mathrm{C}, 250 \mathrm{Watt}\right)$, all values refer to the isolated yields. ${ }^{b}$ Traces of the starting materials were detected by TLC.

An alternate Pd-free synthesis of 2-methyl-4-(5-phenythiophen-2-yl)-1,3-thiazole (11).

2-Methyl-4-(5-phenythiophen-2-yl)-1,3-thiazole (11) that was synthesized above, was alternatively prepared by a palladium-free conventional chemical method. Thus, bromination of 2-acetyl-5-phenylthiophene (4) following the literature procedure ${ }^{45}$ yielded 2-(bromoacetyl)-5phenylthiophene (16) in $92 \%$ yield. The latter was then treated with thioacetamide in absolute ethanol at reflux to give 2-methyl-4-(5-phenythiophen-2-yl)-1,3-thiazole (11) in 95\% yield as shown in Scheme 2.

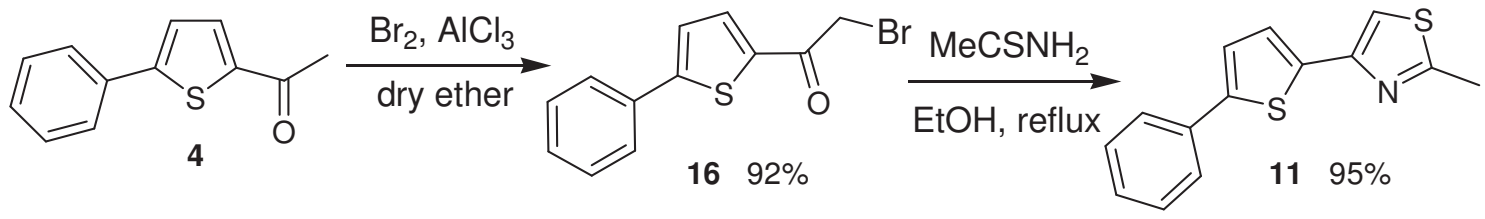

Scheme 2. Alternative Synthesis of 2-methyl-4-(5-phenythiophen-2-yl)-1,3-thiazole (11).

\section{Conclusions}

Although both the coupling candidates 2-acetyl-5-bromothiophene (2) and 4-(5-bromothiophen2-yl)-2-methyl-1,3-thiazole (10) have a bromine atom attached to the thiophene moiety, the reactivity of the thiazole containing candidate $\mathbf{1 0}$ was sharply different from that of $\mathbf{2}$ in water under microwave irradiation condition. Optimization of the catalytic conditions led to exploring the suitable coupling condition for such deactivated thiazolyl bromides. The obtained findings will be a lead to further investigations for coupling reactions of related deactivated bromides. 


\section{Experimental Section}

General. Melting points were determined in open glass capillaries with a Gallenkamp apparatus and are uncorrected. The infrared spectra were recorded in potassium bromide disks on a PyeUnicam SP 3-300 and Shimadzu FTIR 8101 PC infrared spectrophotometer. NMR spectra were recorded with a Varian Mercury VXR-300 NMR spectrometer at $300 \mathrm{MHz}\left({ }^{1} \mathrm{H}\right.$ NMR) and at $75.46 \mathrm{MHz}\left({ }^{13} \mathrm{C} \mathrm{NMR}\right)$ using deuterated chloroform $\left(\mathrm{CDCl}_{3}\right)$ or dimethylsulphoxide (DMSO- $\left.d_{6}\right)$. Chemical shifts are quoted in $\delta$ and were related to that of the solvents. Mass spectra (EI) were obtained at $70 \mathrm{eV}$ with a type Shimadzu GCMQP 1000 EX spectrometer. Microwave experiments were carried out using a CEM Discover Labmate ${ }^{\mathrm{TM}}$ microwave apparatus (300 W with ChemDriver ${ }^{\mathrm{TM}}$ Software). Synthesis of the Pd(II)-complex 1, ${ }^{36}$-acetyl-5-bromothiophene (2), ${ }^{40,41} 5$-bromo-2-(bromoacetyl)thiophene (9), ${ }^{44}$ and 2-(bromoacetyl)-5-phenylthiophene (16) ${ }^{45}$ were accomplished following the procedures reported in literature.

Synthesis of 4-(5-bromothiophen-2-yl)-2-methyl-1,3-thiazole (10). To a solution of 5-bromo2-(bromoacetyl)thiophene (9) $(9.9 \mathrm{~g}, 35 \mathrm{mmol})$ in absolute ethanol $(20 \mathrm{~mL})$, thioacetamide $(2.62$ $\mathrm{g}, 35 \mathrm{mmol}$ ) was added and the mixture was refluxed for $2 \mathrm{hr}$ then left to cool to room temperature and finally poured onto cold aqueous ammonia $(\mathrm{pH} \mathrm{10).} \mathrm{The} \mathrm{solid} \mathrm{product} \mathrm{so}$ formed was filtered off, washed with water and dried. Recrystallization from ethanol afforded an off-white solid of 4-(5-bromothiophen-2-yl)-2-methyl-1,3-thiazole (10) $(7.1 \mathrm{~g}, 78 \%$ yield). mp 76-78 ${ }^{\circ}$; IR $\left(v_{\max }, \mathrm{cm}^{-1}\right): 3085,2930,1610,1440 .{ }^{1} \mathrm{H}$ NMR (DMSO- $\left.d_{6}\right) \delta 2.66\left(\mathrm{~s}, 3 \mathrm{H}, \mathrm{CH}_{3}\right)$, $7.20(\mathrm{~d}, 1 \mathrm{H}, J 3.9 \mathrm{~Hz}), 7.36(\mathrm{~d}, 1 \mathrm{H}, J 3.9 \mathrm{~Hz}), 7.79(\mathrm{~s}, 1 \mathrm{H}) ;{ }^{13} \mathrm{C}$ NMR (DMSO- $\left.d_{6}\right) \delta_{\mathrm{c}} 18.6,110.9$, 112.7, 124.2, 131.3, 139.7, 147.3, 166.2; MS m/z (\%) $261\left(\mathrm{M}^{+}+2,42.1\right), 259\left(\mathrm{M}^{+}, 53.7\right), 138$ (58.1), 94 (66.8), 93 (52.9), 81 (47.3), 68 (100). Anal. Calcd for $\mathrm{C}_{8} \mathrm{H}_{6} \mathrm{BrNS}_{2}$ : C, 36.93; H, 2.32; N, 5.38; S, 24.65. Found: C, 36.56; H, 2.40; N, 5.32; S, 24.81\%.

Suzuki-Miyaura coupling of 2-acetyl-5-bromothiophene (2) with arylboronic acids using Pd-complex 1 in water under thermal heating. General Procedure. A mixture of 2-acetyl-5bromothiophene (2) $(205 \mathrm{mg}, 1 \mathrm{mmol})$ and the appropriate arylboronic acids 3a-e (1.2 mmol), TBAB (194 mg, $0.6 \mathrm{mmol}$ ), palladium complex 1 (1.14 mg, $0.25 \mathrm{~mol} \%)$ KOH (112 mg, 2 $\mathrm{mmol})$, and water $(3 \mathrm{~mL})$ were heated under reflux for the appropriate reaction times as listed in Table 1 (monitored by TLC). The products were then extracted with ethylacetate (EtOAc) (3x20 $\mathrm{mL}$ ). The combined organic extracts were dried over anhydrous $\mathrm{MgSO}_{4}$ then filtered and the solvent was evaporated under reduced pressure. The residue was then subjected to separation via flash column chromatography with n-hexane/EtOAc (4:1) as an eluent to give the corresponding pure cross-coupled products $\mathbf{4 - 8}$.

Suzuki-Miyaura coupling of 2-acetyl-5-bromothiophene (2) with arylboronic acids using Pd-complex 1 in water under microwave irradiation. General Procedure. 2-Acetyl-5bromothiophene (2) $(205 \mathrm{mg}, 1 \mathrm{mmol})$ and arylboronic acids 3a-e (1.2 mmol), TBAB (194 mg, 
$0.6 \mathrm{mmol})$, palladium complex 1 (1.2 $\mathrm{mg}, 0.25 \mathrm{~mol} \%), \mathrm{KOH}$ (112 mg, $2 \mathrm{mmol})$, and distilled water $(3 \mathrm{~mL})$ were mixed in a process glass vial. The vial was capped properly, and thereafter the mixture was heated under microwave irradiating conditions at $160^{\circ} \mathrm{C}$ and 250 Watt for the appropriate reaction time, as listed in Table 1. After the reaction was almost complete, the crosscoupled products were extracted and then subjected to separation via flash column chromatography as above to give the corresponding pure cross-coupled products 4-8.

2-Acetyl-5-phenylthiophene (4). Yellow solid, mp 113-114 ${ }^{\circ} \mathrm{C}$ (Lit. $\left.{ }^{27} \mathrm{mp} \mathrm{109-110}{ }^{\circ} \mathrm{C}\right)$; IR ( $v_{\max }$, $\left.\mathrm{cm}^{-1}\right): 1647,1442,1353,1276,756 .{ }^{1} \mathrm{H} \mathrm{NMR}\left(\mathrm{CDCl}_{3}\right) \delta_{\mathrm{H}} 2.41\left(3 \mathrm{H}, \mathrm{s}, \mathrm{COCH}_{3}\right), 7.15\left(1 \mathrm{H}_{\text {thiophene, }}\right.$ $\left.\mathrm{d},{ }^{3} J_{\mathrm{HH}} 4.2 \mathrm{~Hz}, 1 \mathrm{CH}\right), 7.21-7.23\left(3 \mathrm{H}_{\mathrm{arom}}, \mathrm{m}, 3 \mathrm{CH}\right), 7.45\left(1 \mathrm{H}_{\text {arom }}, \mathrm{d},{ }^{3} J_{\mathrm{HH}} 6.6 \mathrm{~Hz}, 1 \mathrm{CH}\right), 7.52$ $\left(1 \mathrm{H}_{\text {thiophene, }} \mathrm{d},{ }^{3} \mathrm{~J}_{\mathrm{HH}} 4.2 \mathrm{~Hz}, 1 \mathrm{CH}\right)$; MS (EI, $\left.70 \mathrm{eV}\right): \mathrm{m} / z(\%) 202\left(\mathrm{M}^{+}, 68.2\right), 191$ (37.6), 162 (11.5), 92 (56.2), 84 (35.3), 76 (54.5), 50 (100).

2-Acetyl-5-(4-chlorophenyl)thiophene (5). Greenish-yellow solid, mp 135-136 ${ }^{\circ} \mathrm{C}$ (Lit. ${ }^{21} \mathrm{mp}$ 114-115 $\left.{ }^{\circ} \mathrm{C}\right)$; IR $\left(v_{\max }, \mathrm{cm}^{-1}\right): 2962,2873,1647,1442,1276,1099 .{ }^{1} \mathrm{H}$ NMR (DMSO- $\left.d_{6}\right) \delta_{\mathrm{H}} 2.55$ $\left(3 \mathrm{H}, \mathrm{s}, \mathrm{COCH}_{3}\right), 7.52\left(2 \mathrm{H}_{\text {arom }}, \mathrm{d},{ }^{3} J_{\mathrm{HH}} 7.7 \mathrm{~Hz}, 2 \mathrm{CH}\right), 7.66\left(1 \mathrm{H}_{\text {thiophene, }},{ }^{3},{ }^{3} J_{\mathrm{HH}} 3.9 \mathrm{~Hz}, 1 \mathrm{CH}\right), 7.79$ $\left(2 \mathrm{H}_{\text {arom }}, \mathrm{d},{ }^{3} J_{\mathrm{HH}} 7.5 \mathrm{~Hz}, 2 \mathrm{CH}\right), 7.94\left(1 \mathrm{H}_{\text {thiophene, }} \mathrm{d},{ }^{3} \mathrm{~J}_{\mathrm{HH}} 3.9 \mathrm{~Hz}, 1 \mathrm{CH}\right) ; \mathrm{MS}(\mathrm{EI}, 70 \mathrm{eV}): \mathrm{m} / \mathrm{z}(\%)$ $238\left(\mathrm{M}^{+}+2,77.7\right), 236\left(\mathrm{M}^{+}, 100\right), 193$ (13.1), 151 (65.3), 149 (98.4), 114 (68.6), 78 (48.5).

2-Acetyl-5-(4-methoxyphenyl)thiophene (6). Pale yellow solid, mp 160-161 ${ }^{\circ} \mathrm{C}$ (Lit. ${ }^{22} \mathrm{mp} 156-$ $\left.157{ }^{\circ} \mathrm{C}\right)$; IR $\left(v_{\max }, \mathrm{cm}^{-1}\right): 1643,1442,1249,1022 .{ }^{1} \mathrm{H}$ NMR (DMSO- $\left.d_{6}\right): \delta_{\mathrm{H}} 2.49\left(3 \mathrm{H}, \mathrm{s}, \mathrm{COCH}_{3}\right)$, $3.80\left(3 \mathrm{H}, \mathrm{s}, \mathrm{OCH}_{3}\right), 7.02\left(2 \mathrm{H}_{\mathrm{arom}}, \mathrm{d},{ }^{3} J_{\mathrm{HH}} 7.5 \mathrm{~Hz}, 2 \mathrm{CH}\right), 7.52\left(1 \mathrm{H}_{\text {thiophene, }} \mathrm{d},{ }^{3} J_{\mathrm{HH}} 5.7 \mathrm{~Hz}, 1 \mathrm{CH}\right)$, $7.71\left(2 \mathrm{H}_{\text {arom }}, \mathrm{d},{ }^{3} J_{\mathrm{HH}} 7.5 \mathrm{~Hz}, 2 \mathrm{CH}\right), 7.91\left(1 \mathrm{H}_{\text {thiophene, }} \mathrm{d},{ }^{3} J_{\mathrm{HH}} 5.7 \mathrm{~Hz}, 1 \mathrm{CH}\right),{ }^{13} \mathrm{C}$ NMR (DMSO- $\left.d_{6}\right)$ : $\delta_{\mathrm{c}}$ 26.2, 55.3, 114.6, 123.6, 127.4, 135.1, 141.6, 160.0, 190; MS (EI, $\left.70 \mathrm{eV}\right): \mathrm{m} / \mathrm{z}(\%) 232\left(\mathrm{M}^{+}\right.$, 100), 202 (13.5) 189 (33.1), 145 (91.8), 114 (25.4), 102 (48.7), 93 (20.2), 74 (32.7).

2-Acetyl-5-(4-methylphenyl)thiophene (7). Off-white solid, mp 118-119 ${ }^{\circ} \mathrm{C}$ (Lit. ${ }^{21} \mathrm{mp} 115-116$ $\left.{ }^{\circ} \mathrm{C}\right)$; IR $\left(v_{\max }, \mathrm{cm}^{-1}\right): 1647,1442,1280,1029,798 .{ }^{1} \mathrm{H}$ NMR (DMSO- $\left.d_{6}\right): \delta_{\mathrm{H}} 2.34\left(3 \mathrm{H}, \mathrm{s}, \mathrm{CH}_{3}\right)$, $2.55\left(3 \mathrm{H}, \mathrm{s}, \mathrm{COCH}_{3}\right), 7.26\left(2 \mathrm{H}_{\mathrm{arom}}, \mathrm{d},{ }^{3} J_{\mathrm{HH}} 7.8 \mathrm{~Hz}, 2 \mathrm{CH}\right), 7.58\left(1 \mathrm{H}_{\text {thiophene }}, \mathrm{d},{ }^{3} J_{\mathrm{HH}} 4.2 \mathrm{~Hz}, 1 \mathrm{CH}\right)$, $7.63\left(2 \mathrm{H}_{\text {arom }}, \mathrm{d},{ }^{3} J_{\mathrm{HH}} 7.8 \mathrm{~Hz}, 2 \mathrm{CH}\right), 7.91\left(1 \mathrm{H}_{\text {thiophene, }} \mathrm{d},{ }^{3} J_{\mathrm{HH}} 4.2 \mathrm{~Hz}, 1 \mathrm{CH}\right) ;{ }^{13} \mathrm{C}$ NMR (DMSO- $\left.d_{6}\right)$ : $\delta_{\mathrm{c}} 20.8,26.3,120.5,124.3,125.8,129.9,135.0,138.8,142.1,151.6,190.4 ; \mathrm{MS}$ (EI, $\left.70 \mathrm{eV}\right): \mathrm{m} / \mathrm{z}$ (\%) 216 (M+ 85.7), 201 (100), 171 (11.9), 128 (98.3), 114 (22.1), 100 (25.7), 85 (17.5).

2-Acetyl-3 ,5-bithiophene (8). Pale green solid, mp 122-123 ${ }^{\circ} \mathrm{C}$ (Lit. ${ }^{23} \mathrm{mp} \mathrm{119-120}{ }^{\circ} \mathrm{C}$ ); IR $\left(v_{\max }, \mathrm{cm}^{-1}\right): 3097,1655,1450,1276,1029 .{ }^{1} \mathrm{H}$ NMR $\left(\mathrm{DMSO}-d_{6}\right): \delta_{\mathrm{H}} 2.5\left(3 \mathrm{H}, \mathrm{s}, \mathrm{COCH}_{3}\right), 7.52$ $\left(1 \mathrm{H}_{\text {thiophene }}, \mathrm{d},{ }^{3} J_{\mathrm{HH}} 4.2 \mathrm{~Hz}, 1 \mathrm{CH}\right), 7.67-7.70\left(2 \mathrm{H}_{\text {arom }}, \mathrm{m}, 2 \mathrm{CH}\right), 7.69\left(1 \mathrm{H}_{\text {thiophene }}, \mathrm{d},{ }^{3} J_{\mathrm{HH}} 4.2 \mathrm{~Hz}\right.$, $1 \mathrm{CH}), 8.0\left(1 \mathrm{H}_{\text {thiophene, }} \mathrm{s}, 1 \mathrm{CH}\right) ;{ }^{13} \mathrm{C} \mathrm{NMR}\left(\mathrm{DMSO}-d_{6}\right): \delta_{\mathrm{c}} 26.2,122.9,124.8,125.9,127.9,134.1$, 134.9, 141.7, 146.3, 190.4; MS (EI, $70 \mathrm{eV}): m / z(\%) 208\left(\mathrm{M}^{+}, 100\right), 165$ (24.2), 121 (76.3), 96 (26.8), 82 (33.1), 68 (21.4).

Effect of base, solvent and Pd-complex 1 concentration on Suzuki coupling of 4-(5bromothiophen-2-yl)-2-methyl-1,3-thiazole (10) with phenylboronic acid (3a) under microwave irradiation.

A mixture of 4-(5-bromothiophen-2-yl)-2-methyl-1,3-thiazole (10) (266 mg, 1 mmol), phenylboronic acid (3a) (146 mg, $1.2 \mathrm{mmol})$, TBAB (194 mg, $0.6 \mathrm{mmol})$, palladium complex 1 $(0.6 \mathrm{mg}, 0.125 \mathrm{~mol} \%)$ and $\mathrm{KOH}(112 \mathrm{mg}, 2 \mathrm{mmol})$, in water $(3 \mathrm{~mL})$ was subjected to microwave 
irradiation at $160^{\circ} \mathrm{C}$ and 250 Watt for 30 minutes to give 4-(5-phenylthiophen-2-yl)-2-methyl1,3-thiazole (11). The same experiment was repeated using palladium complex $\mathbf{1}$ in different concentration $(0.25,0.5$, and $1 \mathrm{~mol} \%)$ with respect to 4-(5-bromothiophen-2-yl)-2-methyl-1,3thiazole (11) using different solvents and bases. The molar ratio of the reaction components were in all cases as follows; 4-(5-bromothiophen-2-yl)-2-methyl-1,3-thiazole, phenylboronic acid, TBAB, base, solvent 1 / 1.2 / 0.6 / 2 / $3 \mathrm{~mL}$. The results of this study are outlined in Table 2.

Suzuki-Miyaura coupling of 4-(5-bromothiophen-2-yl)-2-methyl-1,3-thiazole (10) with phenylboronic acid 3a under thermal heating.

A mixture of 4-(5-bromothiophen-2-yl)-2-methyl-1,3-thiazole (10) (266 mg, $1 \mathrm{mmol}$ ) and phenylboronic acid 3a (146 mg, $1.2 \mathrm{mmol})$, TBAB (194 mg, $0.6 \mathrm{mmol}$ ), palladium complex 1 (4.6 mg, $1 \mathrm{~mol} \%$ ) and $\mathrm{Cs}_{2} \mathrm{CO}_{3}(652 \mathrm{mg}, 2 \mathrm{mmol})$, in DMF (3 mL) were refluxed at $100{ }^{\circ} \mathrm{C}$ for 40 hours as listed in Table 2. The starting bromide 4-(5-bromothiophen-2-yl)-2-methyl-1,3-thiazole (10) was almost completely recovered without reaction.

Suzuki-Miyaura cross-coupling of 4-(5-bromothiophen-2-yl)-2-methyl-1,3-thiazole (10) with arylboronic acids 3a-e under microwave irradiation.

General Procedure: 4-(5-Bromothiophen-2-yl)-2-methyl-1,3-thiazole (10) (266 mg, $1 \mathrm{mmol}$ ) and the appropriate arylboronic acids 3a-e $(1.2 \mathrm{mmol})$, TBAB (194 $\mathrm{mg}, 0.6 \mathrm{mmol})$, palladium complex 1 (4.6 mg, $1 \mathrm{~mol} \%), \mathrm{Cs}_{2} \mathrm{CO}_{3}(652 \mathrm{mg}, 2 \mathrm{mmol})$, and DMF (3 mL) were mixed in a process glass vial. The vial was capped properly, and thereafter the mixture was heated under microwave irradiating conditions at $160{ }^{\circ} \mathrm{C}$ and 250 Watt for the appropriate reaction time as listed in Table 3. After the reaction was almost complete the cross-coupled products were then extracted with EtOAc $(3 \times 20 \mathrm{~mL})$. The combined organic extracts were dried over anhydrous $\mathrm{MgSO}_{4}$ then filtered and the solvent was evaporated under reduced pressure. The residue was then subjected to separation via flash column chromatography with n-hexane/EtOAc (4:1) as an eluent to give the corresponding pure cross-coupled products 11-15. The results of this study are outlined in Table 3.

4-(5-Phenylthiophen-2-yl)-2-methyl-1,3-thiazole (11). Yellow powder, mp $92-93{ }^{\circ} \mathrm{C}$; IR $\left(v_{\max }\right.$, $\left.\mathrm{cm}^{-1}\right): 3093,2933,1610,1440,1238 .{ }^{1} \mathrm{H} \mathrm{NMR}\left(\mathrm{CDCl}_{3}\right): \delta_{\mathrm{H}} 2.78\left(3 \mathrm{H}, \mathrm{s}, \mathrm{CH}_{3}\right) 7.21\left(1 \mathrm{H}_{\text {thiazole }}, \mathrm{s}\right.$, $1 \mathrm{CH}), 7.27-7.41\left(5 \mathrm{H}_{\text {arom+thiophene, }} \mathrm{m}, 5 \mathrm{CH}\right), 7.64\left(2 \mathrm{H}_{\text {arom }}, \mathrm{d},{ }^{3} J_{\mathrm{HH}} 7.5 \mathrm{~Hz}, 2 \mathrm{CH}\right) ;{ }^{13} \mathrm{C} \mathrm{NMR}$ $\left(\mathrm{CDCl}_{3}\right): \delta_{\mathrm{c}} 19.2,110.8,123.7,124.9,125.6,126.2,127.5,128.9,129.1,133.4,137.3,143.8$, 149.3, 166.3; MS (EI, $70 \mathrm{eV}): \mathrm{m} / z$ (\%) $257\left(\mathrm{M}^{+}, 85\right), 180$ (40.3), 148 (22.6), 123 (26.5), 111 (53.9), 95 (43.6). Anal. Calcd for $\mathrm{C}_{14} \mathrm{H}_{11} \mathrm{NS}_{2}$ : C, 65.33; H, 4.31; N, 5.44; S, 24.92\%. Found: C, $65.11 ; \mathrm{H}, 3.89 ; \mathrm{N}, 5.22 ; \mathrm{S}, 25.07 \%$.

4-(5-(4-Chlorophenyl)thiophen-2-yl)-2-methylthiazole (12). Brown solid, mp 118-120 ${ }^{\circ} \mathrm{C}$; IR $\left(v_{\max }, \mathrm{cm}^{-1}\right): 3105,1442,1272,1091,794 .{ }^{1} \mathrm{H}$ NMR $\left(\mathrm{CDCl}_{3}\right): \delta_{\mathrm{H}} 2.58\left(3 \mathrm{H}, \mathrm{s}, \mathrm{CH}_{3}\right), 7.23$ $\left(1 \mathrm{H}_{\text {thiazole }}, \mathrm{s}, 1 \mathrm{CH}\right), 7.29\left(1 \mathrm{H}_{\text {thiophene, }} \mathrm{d},{ }^{3} \mathrm{~J}_{\mathrm{HH}} 3.9 \mathrm{~Hz}, 1 \mathrm{CH}\right), 7.40\left(2 \mathrm{H}_{\text {arom }}, \mathrm{d},{ }^{3} J_{\mathrm{HH}} 7.8 \mathrm{~Hz}, 2 \mathrm{CH}\right)$, $7.58\left(2 \mathrm{H}_{\text {arom }}, \mathrm{d},{ }^{3} J_{\mathrm{HH}} 7.8 \mathrm{~Hz}, 2 \mathrm{CH}\right), 7.65\left(1 \mathrm{H}_{\text {thiophene, }} \mathrm{d},{ }^{3} J_{\mathrm{HH}} 3.9 \mathrm{~Hz}, 1 \mathrm{CH}\right) ;{ }^{13} \mathrm{C} \mathrm{NMR}\left(\mathrm{CDCl}_{3}\right): \delta_{\mathrm{c}}$ 26.5, 124.1, 125.9, 162.7, 127.4, 129, 129.3, 131.8, 133.1, 133.3, 134.9, 143.4, 151.1, 190.4; MS (EI, $70 \mathrm{eV}): m / z(\%) 293\left(\mathrm{M}^{+}+2,23.2\right), 291\left(\mathrm{M}^{+}, 51.9\right), 221$ (100), 148 (69.1), 113 (33.6), 85 
(21.3). Anal. Calcd for $\mathrm{C}_{14} \mathrm{H}_{10} \mathrm{ClNS}_{2}$ : C, 57.62; H, 3.45; N, 4.80; S, 21.98\%. Found: C, 57.42; H, $3.64 ; \mathrm{N}, 4.76 ; \mathrm{S}, 21.68 \%$.

4-(5-(4-Methoxyphenyl)thiophen-2-yl)-2-methylthiazole (13). Grey powder, mp 122-124 ${ }^{\circ} \mathrm{C}$; IR $\left(v_{\max }, \mathrm{cm}^{-1}\right): 3097,2931,1604,1434,1249,1180,802 .{ }^{1} \mathrm{H}$ NMR $\left(\mathrm{CDCl}_{3}\right): \delta_{\mathrm{H}} 2.77(3 \mathrm{H}, \mathrm{s}$, $\left.\mathrm{CH}_{3}\right), 3.84\left(3 \mathrm{H}, \mathrm{s}, \mathrm{CH}_{3} \mathrm{O}\right), 6.92\left(2 \mathrm{H}_{\text {arom }}, \mathrm{d},{ }^{3} J_{\mathrm{HH}} 8.1 \mathrm{~Hz}, 2 \mathrm{CH}\right), 7.15\left(1 \mathrm{H}_{\text {thiophene, }} \mathrm{d},{ }^{3} J_{\mathrm{HH}} 3.9 \mathrm{~Hz}\right.$, $1 \mathrm{CH}), 7.27(\mathrm{~s}, 1 \mathrm{H}), 7.35\left(1 \mathrm{H}_{\text {thiophene, }} \mathrm{d},{ }^{3} J_{\mathrm{HH}} 3.6 \mathrm{~Hz}, 1 \mathrm{CH}\right), 7.56\left(2 \mathrm{H}_{\text {arom }}, \mathrm{d},{ }^{3} J_{\mathrm{HH}} 8.1 \mathrm{~Hz}, 2 \mathrm{CH}\right)$; ${ }^{13} \mathrm{C} \mathrm{NMR}\left(\mathrm{CDCl}_{3}\right): \delta_{\mathrm{c}} 19.2,55.4,110.5,113.9,114.3,122.6,124.7,126.9,127.7,130.1,136.5$, 143.7, 149.5, 159.3, 166.1; MS (EI, $70 \mathrm{eV}): m / z(\%) 287\left(\mathrm{M}^{+}, 31.5\right), 272$ (19.1), 257 (34), 255 (100), 128 (37.2), 95 (41.6), 64 (88.2). Anal. Calcd for $\mathrm{C}_{15} \mathrm{H}_{13} \mathrm{NOS}_{2}$ : C, 62.69; H, 4.56; N, 4.87; S, 22.31\%. Found: C, 61.63; H, 4.36; N, 4.51; S, $22.58 \%$.

2-Methyl-4-(5-(4-tolyl)thiophen-2-yl)thiazole (14). Grey powder, mp $114-116{ }^{\circ} \mathrm{C}$; IR $\left(v_{\max }, \mathrm{cm}^{-}\right.$ $\left.{ }^{1}\right)$ : 3093, 2916, 1496, 1438, 1284, 1161. ${ }^{1} \mathrm{H} \mathrm{NMR}\left(\mathrm{CDCl}_{3}\right): \delta_{\mathrm{H}} 2.37\left(3 \mathrm{H}, \mathrm{s}, \mathrm{CH}_{3}\right), 2.76(3 \mathrm{H}, \mathrm{s}$, $\left.\mathrm{COCH}_{3}\right), 7.18-7.26\left(4 \mathrm{H}_{\text {arom }}, \mathrm{m}, 4 \mathrm{CH}\right), 7.37\left(1 \mathrm{H}_{\text {thiophene, }} \mathrm{d},{ }^{3} J_{\mathrm{HH}} 3.9 \mathrm{~Hz}, 1 \mathrm{CH}\right), 7.53\left(2 \mathrm{H}_{\text {arom }}, \mathrm{d}\right.$, $\left.{ }^{3} J_{\mathrm{HH}} 7.8 \mathrm{~Hz}, 2 \mathrm{CH}\right) ;{ }^{13} \mathrm{C} \mathrm{NMR}\left(\mathrm{CDCl}_{3}\right): \delta_{\mathrm{c}} 19.2,21.2,110.6,123.1,124.6,125.5,126.1,129.5$, 129.7, 131.5, 136.9, 137.4, 143.9, 149.5, 166; MS (EI, $70 \mathrm{eV}): \mathrm{m} / \mathrm{z}$ (\%) $271\left(\mathrm{M}^{+}, 20.6\right), 270$ (100), 230 (26.8), 129 (10.4), 93 (5.2), 83 (5.6). Anal. Calcd for $\mathrm{C}_{15} \mathrm{H}_{13} \mathrm{NS}_{2}$ : C, 66.38; H, 4.83; N, 5.16; S, 23.63\%. Found: C, 67.03; H, 4.62; N, 5.01; S, 23.84\%.

Alternative synthesis of 2-methyl-4-(5-phenylthiophen-2-yl)-1,3-thiazole (11). To a solution of 2-(bromoacetyl)-5-phenylthiophene (16) (2.46 g, $9 \mathrm{mmol})$ in absolute ethanol (20 $\mathrm{mL})$, thioacetamide $(0.66 \mathrm{~g}, 9 \mathrm{mmol})$ was added. The reaction mixture was refluxed for two hours, left to cool to room temperature then poured onto cold aqueous ammonia solution ( $\mathrm{pH} 10$ ). The solid product that formed was filtered off, washed with water and dried. Recrystallization from ethanol afforded 2-methyl-4-(5-phenylthiophen-2-yl)-1,3-thiazole (11) in $2.2 \mathrm{~g}$ (95\% yield). The obtained product was in complete agreement ( $\mathrm{mp}$ and spectral data) with the product that obtained above via Suzuki coupling reaction (Table 3, run 1).

\section{Supplementary Material}

Supplemetary material is available.

\section{References}

1. Swanston, J. Thiophene In Ullmann's Encyclopedia of Industrial Chemistry Wiley-VCH: Weinheim, 2006.

2. Stahl, S. Stahl's Essential Psychopharmacology: Prescriber's Guide. Cambridge University Press: New York, NY, 2009; pp 523.

3. Chao, E. C. Drug Fut. 2011, 36, 351. 
http://dx.doi.org/10.1358/dof.2011.036.05.1590789

4. Russel, K. R.; Press, B. J.; Rampulla, A. R. J. Med. Chem. 1988, 31, 1786.

http://dx.doi.org/10.1021/jm00117a019

5. Kaur, H.; Kumar, S.; Sing, I.; Saxena, K. K.; Kumar, A. Dig. J. Nanomater. Bios. 2010, 5, 67.

6. Badar, S. M. I. Turk. J. Chem. 2001, 35, 131.

7. Sigmundova, I.; Zahradnik, P.; Magdolen, P.; Bujdakova, H. Arkivoc 2008, (viii), 183. http://dx.doi.org/10.3998/ark.5550190.0009.815

8. Alonso, F., Beletskaya, I. P., Yus, M. Tetrahedron 2008, 64, 3047. http://dx.doi.org/10.1016/j.tet.2007.12.036

9. Bellina, F., Carpita, A., Rossi, R. Synthesis 2004, 15, 2419.

10. Miyaura, N. J. Organomet. Chem. 2002, 653, 54. http://dx.doi.org/10.1016/S0022-328X(02)01264-0

11. Suzuki, A. J. Organomet. Chem. 2002, 653, 83. http://dx.doi.org/10.1016/S0022-328X(02)01269-X

12. Kelly, T. R.; Fu, Y.; Sieglen, J. T.; de Silva, H. Org. Lett. 2000, 2, 2351. http://dx.doi.org/10.1021/o1006127a

13. Jeong, S. J.; Higuchi, R.; Miyamoto, T.; Ono, M.; Kuwano, M.; Mawatari, S. F. J. Nat. Prod. 2002, 65, 1344. http://dx.doi.org/10.1021/np010577+

14. Ankita, C.; Jha, K.; Sachin, K. J. Adv. Sci. 2012, 3, 3.

15. Dupont, J.; Consorti, C. S.; Spencer, J. Chem. Rev. 2005, 105, 2527. http://dx.doi.org/10.1021/cr030681r

16. Farina, V. Adv. Synth. Catal. 2004, 346, 1553. http://dx.doi.org/10.1002/adsc.200404178

17. Li, C. J. Chem. Rev. 2005, 105, 3095. http://dx.doi.org/10.1021/cr030009u

18. Hoz, A.; Ortiz, A. D.; Moreno, A. Chem. Soc. Rev. 2005, 34, 164. http://dx.doi.org/10.1039/b411438h

19. Kappe, C. O.; Stadler, A. Microwave Theory, In: Microwaves in Organic and Medicinal Chemistry; Wiley-VCH: Weinhiem, 2005; Chapter 2.

20. Churruca, F.; Hernandez, S.; Perea, M.; SanMartin, R.; Dominguez, E. Chem. Commun. 2013, 49, 1413. http://dx.doi.org/10.1039/c2cc37905h

21. Matiychuk, V. S.; Obushak, N. D.; Lytvyn1, R. Z.; Horak, Yu. I. Chem. Heterocycl. Compds. 2010, 46,50 . http://dx.doi.org/10.1007/s10593-010-0468-3

22. Herbivo, C.; Comel, A.; Kirsch, G.; Manuela, M.; Raposo, M. Tetrahedron 2009, 65, 2079. http://dx.doi.org/10.1016/j.tet.2008.12.078 
23. Molander, G. A.; Biolatto, B. J. Org. Chem. 2003, 68, 4302. http://dx.doi.org/10.1021/jo0342368

24. Kuriyama, M.; Matsuo, S.; Shinozawa, M.; Onomura, O. Org. Lett. 2013, 15, 2716. http://dx.doi.org/10.1021/ol4010189

25. Barrett, M.; Fuchi, N. Jpn. Kokai Tokkyo Koho, JP 2013180955 A 20130912.

26. Molander, G. A.; Trice, S. L. J.; Kennedy, S. M. J. Org. Chem. 2012, 77, 8678. http://dx.doi.org/10.1021/jo301642v

27. Kotha, S.; Kashinath, D.; Lahiri, K.; Sunoj, R. B. Eur. J. Org. Chem. 2004, 4003. http://dx.doi.org/10.1002/ejoc.200400257

28. Bai, L. Chin. Chem. Lett. 2009, 20, 158. http://dx.doi.org/10.1016/j.cclet.2008.10.050

29. Jiang, T.-S.; Wang, G.-W. Adv. Synth. Catal. 2014, 356, 369. http://dx.doi.org/10.1002/adsc.201300843

30. Dawood, K. M.; Kirschning, A. Tetrahedron 2005, 61, 12121. http://dx.doi.org/10.1016/j.tet.2005.07.113

31. Solodenko, W.; Brochwitz, C.; Wartchow, R.; Hashem, Md. A.; Vaultier, M.; Dawood, K. M.; Kirschning, A. Mol. Div. 2005, 9, 333. http://dx.doi.org/10.1007/s11030-005-7457-y

32. Dawood, K. M.; Solodenko, W.; Kirschning, A. Arkivoc 2007, (v), 104.

33. Dawood, K. M. Tetrahedron 2007, 63, 9642. http://dx.doi.org/10.1016/j.tet.2007.07.029

34. Dawood, K. M.; Fayed, M. S.; Elkhalea, M. M. Arkivoc 2009, (xiii), 324.

35. Shaaban, M. R.; Darweesh, A. F.; Dawood, K. M.; Farag, A. F. Arkivoc 2010, (x), 208. http://dx.doi.org/10.3998/ark.5550190.0011.a18

36. Darweesh, A. F.; Shaaban, M. R.; Farag, A. F.; Metz, P.; Dawood, K. M. Synthesis 2010, 3163.

37. Dawood, K. M.; El-Deftar, M. M. Arkivoc 2010, (ix), 319. http://dx.doi.org/10.3998/ark.5550190.0011.930

38. Dawood, K. M.; El-Deftar, M. M. Synthesis 2010, 1030. http://dx.doi.org/10.1055/s-0029-1218662

39. Dawood, K. M.; Farag, A. F.; El-Deftar, M. M. Gardinar, M.; Abdelaziz, H. A. Arkivoc 2013, (iii), 210. http://dx.doi.org/10.3998/ark.5550190.0014.317

40. Smirnov, V. A.; Lipkin, A. E.; Chem. Heterocycl. Compds. 1975, 9, 170. http://dx.doi.org/10.1007/BF00569154

41. Arsenyan, P.; Paegle, E.; Belyakov, S. Tetrahedron Lett. 2010, 51, 205. http://dx.doi.org/10.1016/j.tetlet.2009.10.133

42. Dunleavy, J. K. Platinum Metals Rev. 2006, 50, 110. http://dx.doi.org/10.1595/147106706X111456 
43. Tzschucke, C. C.; Markert, C.; Glatz, H.; Bannwarth, W. Angew. Chem. Int. Ed. 2002, 41, 4500 . http://dx.doi.org/10.1002/1521-3773(20021202)41:23<4500::AID-ANIE4500>3.0.CO;2-N

44. Perez, D. I.; Conde, S.; Pérez, C.; Gil, C.; Simon, D.; Wandosell, F.; Moreno, F. J.; Gelpi, J. L.; Luque, F. J.; Martinez, A. Bioorg. Med. Chem. 2009, 17, 6914. http://dx.doi.org/10.1016/j.bmc.2009.08.042

45. Bagli, J. F.; Mackay, W. D.; Ferdinandi, E.; Cayen, M. N.; Vavra, I.; Pugsley, T.; Lippmann, W. J. Med. Chem. 1976, 19, 876.

http://dx.doi.org/10.1021/jm00229a004 